\title{
Beam loss reduction by injection painting in the 3-GeV rapid cycling synchrotron of the Japan Proton Accelerator Research Complex
}

\author{
H. Hotchi, ${ }^{*}$ H. Harada, N. Hayashi, M. Kinsho, P. K. Saha, Y. Shobuda, F. Tamura, K. Yamamoto, \\ M. Yamamoto, and M. Yoshimoto \\ Japan Atomic Energy Agency (JAEA), Tokai, Naka, Ibaraki, 319-1195, Japan \\ Y. Irie \\ High Energy Accelerator Research Organization (KEK), Tsukuba, Ibaraki, 305-0801, Japan
}

(Received 14 July 2011; published 11 April 2012)

\begin{abstract}
The 3-GeV rapid cycling synchrotron (RCS) of the Japan Proton Accelerator Research Complex was commissioned in October 2007. Via the initial beam tuning and a series of underlying beam studies with low-intensity beams, since December 2009, we have intermittently been performing beam tuning experiments with higher-intensity beams including the injection painting technique. By optimizing the injection painting parameters, we have successfully achieved a $420 \mathrm{~kW}$-equivalent output intensity at a low-level intensity loss of less than $1 \%$. Also the corresponding numerical simulation well reproduced the observed painting parameter dependence on the beam loss, and captured a characteristic behavior of the high-intensity beam in the injection painting process. In this paper, we present the experimental results obtained in the course of the RCS beam power ramp-up, especially on the beam loss reduction achieved by employing the injection painting, together with the numerical simulation results.
\end{abstract}

DOI: 10.1103/PhysRevSTAB.15.040402

PACS numbers: 29.20.dk, 29.27.Ac, 29.27.Fh, 29.27.Bd

\section{INTRODUCTION}

The Japan Proton Accelerator Research Complex (J-PARC) is a multipurpose proton accelerator facility [1], comprising a 400-MeV linac, a 3-GeV rapid cycling synchrotron (RCS), a $50-\mathrm{GeV}$ main ring synchrotron (MR), and three experimental facilities [a materials and life science experimental facility (MLF), a hadron experimental hall, and a neutrino beam line to Kamioka]. In this chain of accelerators, the RCS has two functions as a proton driver to produce pulsed muons and neutrons at the MLF and as an injector to the MR, aiming at $1 \mathrm{MW}$ output beam power.

The J-PARC beam commissioning has proceeded as planned since November 2006 from the linac to the downstream facilities. The RCS was commissioned in October 2007 and made available for user operation in December 2008 with an output beam power of 4-20 kW. By a series of underlying beam tests with such lowintensity beams [2], the output beam power from the RCS in the routine user operation was increased to $120 \mathrm{~kW}$ in December 2009 and to $210 \mathrm{~kW}$ in December 2010. Since then, our efforts have been focused on parameter tuning for higher-intensity beams including the injection painting technique.

\footnotetext{
*hotchi.hideaki@jaea.go.jp

Published by the American Physical Society under the terms of the Creative Commons Attribution 3.0 License. Further distribution of this work must maintain attribution to the author(s) and the published article's title, journal citation, and DOI.
}

The most important issues in achieving such a MW-class high-power beam operation are control and minimization of beam loss to ensure that the machine activation is maintained within the permissible level and to preserve the hands-on-maintenance environment. In high-power proton machines such as the RCS, the space-charge effect especially in the low-energy region is nominated as one of the most crucial sources of beam loss, and it would pose a strong limit on the achievable beam intensity. For this concern, the RCS adopts a multiturn injection painting scheme including the charge-exchange process from negative hydrogen ions $\left(\mathrm{H}^{-}\right)$to protons. This approach permits one to control the charge density distribution of the circulating beam in both the transverse and longitudinal planes for space-charge mitigation and its resultant beam loss reduction particularly in the low-energy region.

Also in the other high-power machines such as the spallation neutron source accumulator ring at the Oak Ridge National Laboratory $[3,4]$ and the ISIS synchrotron at the Rutherford Appleton Laboratory [5,6], the charge density manipulation at injection for both the transverse and longitudinal phase spaces is one of the essential techniques to meet their performance requirements, while the technical skills vary from facility to facility. Therefore, design and beam dynamics studies for injection painting have been actively pursued in various high-power facilities worldwide.

In this paper, we present the results of a series of beam tuning experiments performed using high-intensity beams at the RCS, with particular emphasis on beam loss reduction by injection painting, together with the corresponding numerical simulation results. 


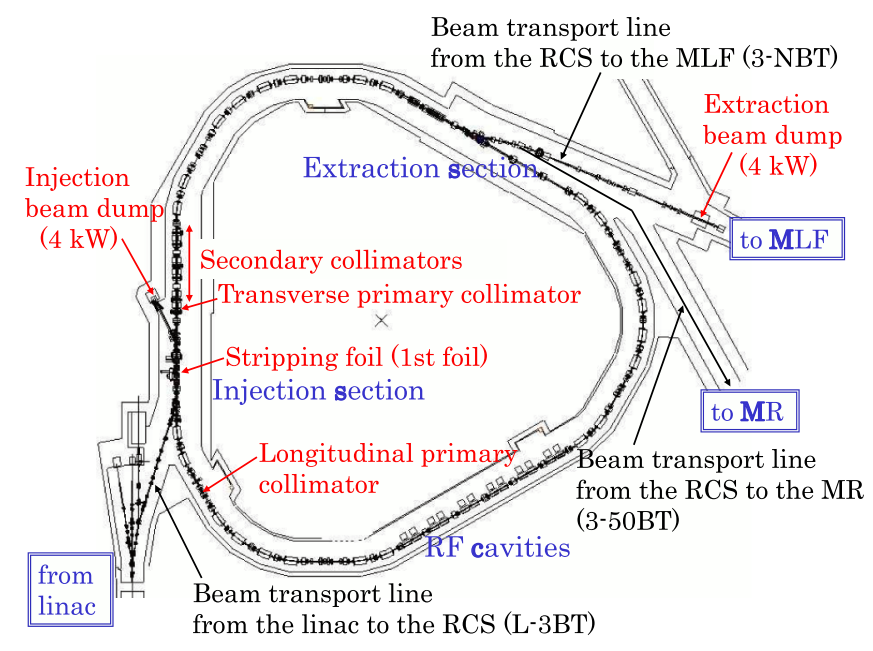

FIG. 1. Schematic view of the RCS.

\section{LAYOUT AND DESIGN PARAMETERS}

Figure 1 shows a schematic view of the RCS, the design parameters of which are listed in Table I. As shown in Fig. $1, \mathrm{a} \mathrm{H}^{-}$beam from the linac is delivered via the
linac-to-3-GeV beam transport line (L-3BT) to the RCS injection point, where it is multiturn charge-exchange injected through a $200 \mu \mathrm{g} / \mathrm{cm}^{2}$-thick hybrid type boronmixed carbon stripping foil (called HBC foil [7]) over a period of $0.5 \mathrm{~ms}$. In order to avoid longitudinal beam loss during the injection, the $\mathrm{H}^{-}$linac beam is equipped with a chopped bunch structure synchronized at the ring $\mathrm{rf}$ frequency $(938 \mathrm{kHz})$ at the injection time. The current injection energy is $181 \mathrm{MeV}$, for which the transverse emittance and momentum spread of the injection beam are designed to be $6 \pi \mathrm{mm}$ mrad (unnormalized) and $\pm 0.1 \%$ in full. The RCS accelerates the injected protons up to $3 \mathrm{GeV}$ at a repetition rate of $25 \mathrm{~Hz}$. Most of the time, the $3-\mathrm{GeV}$ beam from the RCS is transported via the 3-GeV-to-neutron-target beam transport line (3-NBT) to the MLF. A part of the RCS beam (typically four pulses every several seconds) is transported via the $3-\mathrm{GeV}$-to-50$\mathrm{GeV}$ beam transport line (3-50BT) to the MR.

Figure 2 shows the optical functions along the ring. As shown in Figs. 1 and 2, the RCS has a threefold symmetric lattice over its circumference of $348.333 \mathrm{~m}$. Each superperiod consists of two 3-DOFO arc modules and a 3-DOFO

TABLE I. RCS design parameters.

\begin{tabular}{lc}
\hline \hline Circumference & $348.333 \mathrm{~m}$ \\
\hline Superperiodicity & 3 \\
Injection energy & $181 \mathrm{MeV}^{\mathrm{a}}$ \\
Transverse emittance of the injection beam (unnormalized) & $6 \pi \mathrm{mm} \mathrm{mrad}^{\mathrm{b}}$ \\
Momentum spread of the injection beam & $\pm 0.1 \%$ \\
Injection period & $0.5 \mathrm{~ms}(235 \mathrm{turns})^{\mathrm{c}}$ \\
Extraction energy & $3 \mathrm{GeV}$ \\
Repetition rate & $25 \mathrm{~Hz}$ \\
Ramping pattern & $\mathrm{Sinusoidal}$ \\
Harmonic number & 2 \\
Number of bunches & 2 \\
Output beam power & $0.3-0.6 \mathrm{MW}$ \\
Number of particles per pulse & $2.5-5.0 \times 10^{13 \mathrm{e}}$ \\
Ring collimator capability & $4 \mathrm{~kW}$ \\
Betatron tune & $6.45 / 6.42^{\mathrm{f}}$ \\
Natural chromaticity & $-8.5 /-8.8^{\mathrm{g}}$ \\
Transition energy & $9.2 \mathrm{GeV}$ \\
Momentum acceptance & $\pm 1 \%$ \\
Ring acceptance & $486 \pi \mathrm{mm} \mathrm{mrad}$ \\
Collimator acceptance & $324 \pi \mathrm{mm} \mathrm{mrad}$ \\
Transverse painting emittance & $216 \pi \mathrm{mm} \mathrm{mrad}_{\text {Longitudinal beam emittance }}$ \\
Bunching factor at injection & $3.2 \mathrm{eVs}$ \\
Laslett tune shift at injection for $0.3 \mathrm{MW}$ output & 0.4 \\
\hline \hline
\end{tabular}

${ }^{\mathrm{a}}$ It will be upgraded to $400 \mathrm{MeV}$ by adding the ACS linac section.

${ }^{\mathrm{b}}$ It is $4 \pi \mathrm{mm} \mathrm{mrad}$ for $400 \mathrm{MeV}$ injection energy.

'It is $0.5 \mathrm{~ms}$ (307 turns) for $400 \mathrm{MeV}$ injection energy.

${ }^{\mathrm{d}}$ It is $1 \mathrm{MW}$ for $400 \mathrm{MeV}$ injection energy.

${ }^{\mathrm{e}}$ It is $8.3 \times 10^{13}$ for $1 \mathrm{MW}$ output.

${ }^{\mathrm{f}}$ It means horizontal/vertical tunes.

${ }^{\mathrm{g}}$ It means horizontal/vertical chromaticities.

${ }^{\mathrm{h}} \mathrm{It}$ is comparable at $400 \mathrm{MeV}$ injection energy for $1 \mathrm{MW}$ output. 

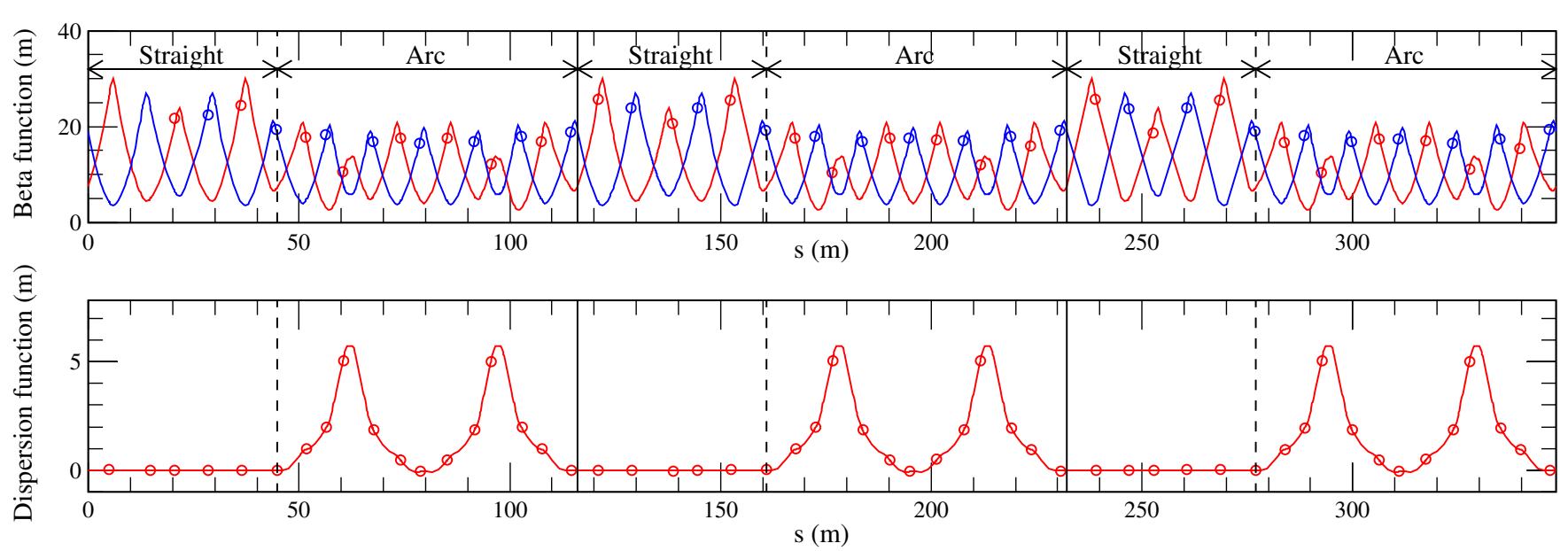

FIG. 2. Beta (top) and dispersion (bottom) functions along the ring, where the red curves are the horizontal ones, the blue curves the vertical ones, and the open circles show the measured results.

long straight insertion, where DOFO describes a defocusing-focusing periodic cell. Each arc module has a missing-bend cell, where the horizontal dispersion has a maximum $(6 \mathrm{~m})$. Such a lattice structure gives a high transition energy $\left(\gamma_{t}=9.2 \mathrm{GeV}\right)$, which is sufficiently far from the extraction energy. This high- $\gamma_{t}$ lattice obtained by the missing-bend technique is a unique feature of the RCS optics design. Three families of sextupole magnets utilized for chromatic correction and a longitudinal primary collimator (scatterer) are installed in the high dispersion areas. On the other hand, the straight insertions have no dispersion. Injection and collimation systems are installed in the first straight section. The injection system uses the first 1.5 cells, while the transverse primary collimator (scatterer) and secondary collimators (absorbers) use the remainder of the cells. Extraction and rf systems are allocated in the second and third straight sections.

The RCS ring acceptance is $486 \pi \mathrm{mm}$ mrad for a possible momentum spread of $\pm 1 \%$, for which the primary collimator aperture is set at $324 \pi \mathrm{mm}$ mrad. Such a large ring acceptance is essential for maintaining the particle loss within the permissible level. Moreover, such a large ratio of the ring acceptance to the collimator aperture (1.5) is necessary for localizing the residual particle loss in the collimator section and minimizing the irradiation of the rest of the ring.

With the current injection energy of $181 \mathrm{MeV}$, the RCS aims at providing more than $300 \mathrm{~kW}$ output beam power. The linac will be upgraded in the 2013 summer maintenance period; the output energy will be improved to $400 \mathrm{MeV}$ with the addition of an annular coupled structure (ACS) linac [8], and the maximum peak current will be increased to $50 \mathrm{~mA}$ (30 $\mathrm{mA}$ at the moment) by replacing the front-end system (ion source and radio-frequency quadrupole linac). After that, the RCS will aim at our final goal of $1 \mathrm{MW}$ output. The above two operations give an equivalent space-charge effect at each injection energy as per the $\beta^{2} \gamma^{3}$ scaling law, where $\beta$ and $\gamma$ are the Lorentz factors. The allowable intensity loss for the $1 \mathrm{MW}$ output operation with $400 \mathrm{MeV}$ injection energy is 3\% at injection, as determined on the basis of the current collimator capability $(4 \mathrm{~kW})$. Therefore, achieving more than $300 \mathrm{~kW}$ output with $181 \mathrm{MeV}$ injection energy at less than 3\% intensity loss will serve as a touchstone for realizing the succeeding $1 \mathrm{MW}$ output with $400 \mathrm{MeV}$ injection energy.

Details of the distinctive features of the RCS devices are described in Ref. [9].

\section{III. $\mathrm{H}^{-}$INJECTION PAINTING IN THE RCS}

In this section, the injection painting technique applied for the transverse and longitudinal phase spaces in the RCS is described.

\section{A. Transverse phase-space painting}

The transverse painting makes use of a controlled phasespace offset between the centroid of the injection beam and the ring closed orbit to form a different particle distribution of the circulating beam from the multiturn injected beam.

Figure 3 shows a schematic view of the RCS injection section. For the beam injection, two types of orbit-bump systems are prepared. One is the shift-bump system (four horizontal pulse dipole magnets; SB1-4) to produce a flattop field during injection, making a horizontal orbitbump offset at the injection point where the stripping foil is installed. The other is the paint-bump system (four horizontal and two vertical pulse dipole magnets; $\mathrm{PBH} 1-4$ and PBV1-2) to make a time dependent bump orbit for beam painting.

In the injection process, the PBHs installed in the ring produce a horizontal closed orbit variation at the injection point by gradual reduction of their field strength. In this manner, the injected beam is filled from the middle to the border of the circulating beam ellipse along its major axis 


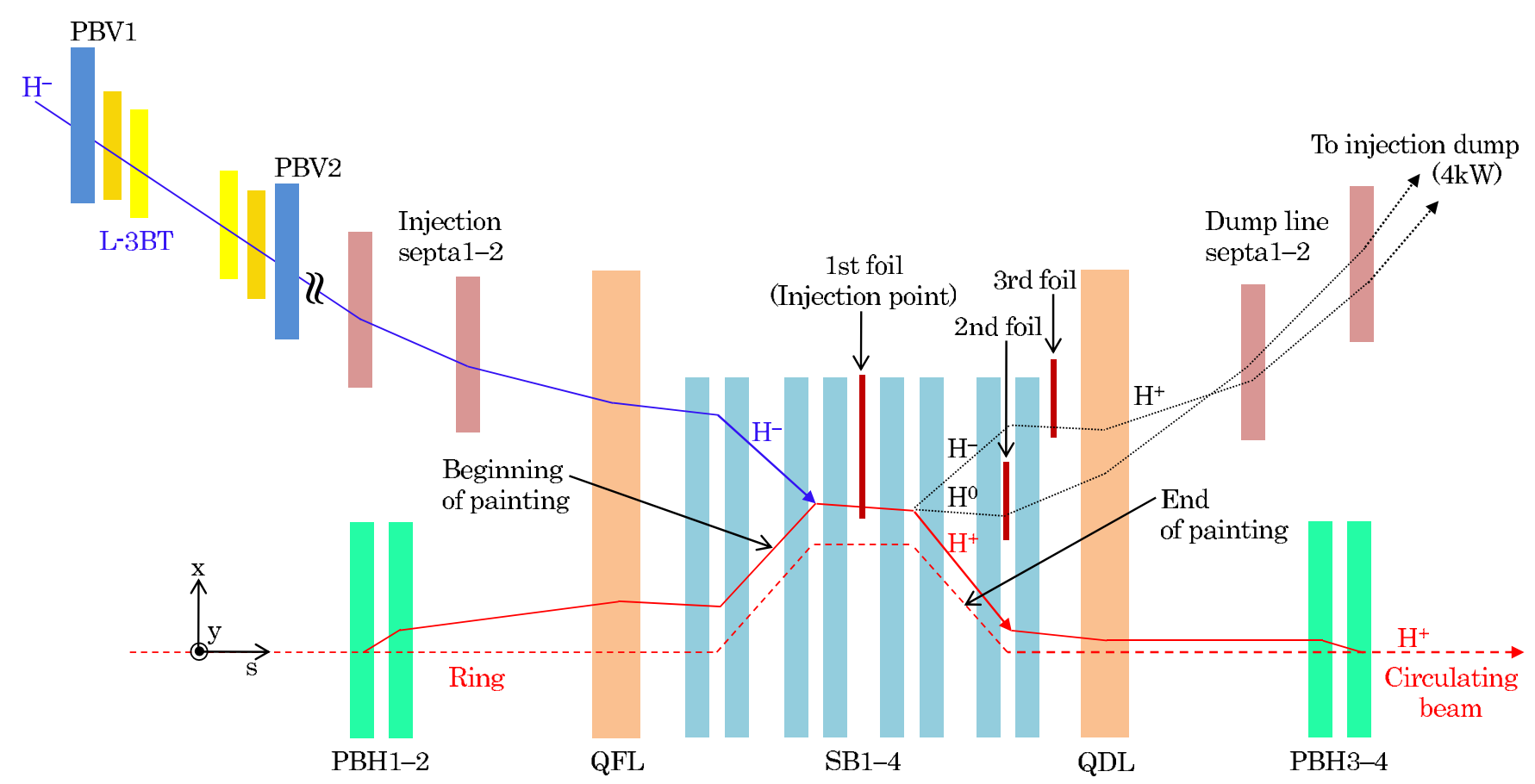

FIG. 3. Schematic view of the RCS injection area.

in the horizontal phase space, as shown in the upper part of Fig. 4. On the other hand, the PBVs, which are installed in the L-3BT nearly 180-degrees upstream of the injection point in betatron phase advance, produce a vertical injection angle change at the injection point. As for the vertical painting, two ways of beam painting processes are available by changing the excitation waveform of the PBVs. One begins at 0 degrees, going to a maximum angle with respect to the ring closed orbit, and the other is the opposite process from the maximum angle to 0 degrees, as shown in the lower part of Fig. 4.

For this painting process, a square-root-type function for the phase-space offset of the injection beam relative to the ring closed orbit was chosen in the present experiment;

$$
x_{\text {paint }}=x_{\max } \sqrt{t / T}, \quad x_{\text {paint }}^{\prime}=-x_{\max }^{\prime} \sqrt{t / T},
$$

for the horizontal plane, and

$$
y_{\text {paint }}=0, \quad y_{\text {paint }}^{\prime}=-y_{\max }^{\prime} \sqrt{t / T},
$$

or

$$
y_{\text {paint }}=0, \quad y_{\text {paint }}^{\prime}=-y_{\max }^{\prime} \sqrt{1-t / T},
$$

for the vertical plane, where $\left(x_{\max }, x_{\max }^{\prime}\right)$ and $\left(y_{\max }, y_{\max }^{\prime}\right)$ are the maximum phase-space offsets for the horizontal and vertical planes corresponding to the border of the circulating beam ellipse with the required painting emittance, $T$ is an injection duration of $0.5 \mathrm{~ms}$, and $t$ is a time step from 0 through the end of injection $T$.

The combination of horizontal painting (1) and vertical painting (2) is known as correlated painting. In this case, the horizontal and vertical emittances of each beam particle $\left(\epsilon_{x}\right.$ and $\left.\epsilon_{y}\right)$ are distributed along $\epsilon_{x} \approx \epsilon_{y}$, producing a rectangular cross section of the circulating beam in the 2-dimensional $x-y$ plane after painting if assuming simple

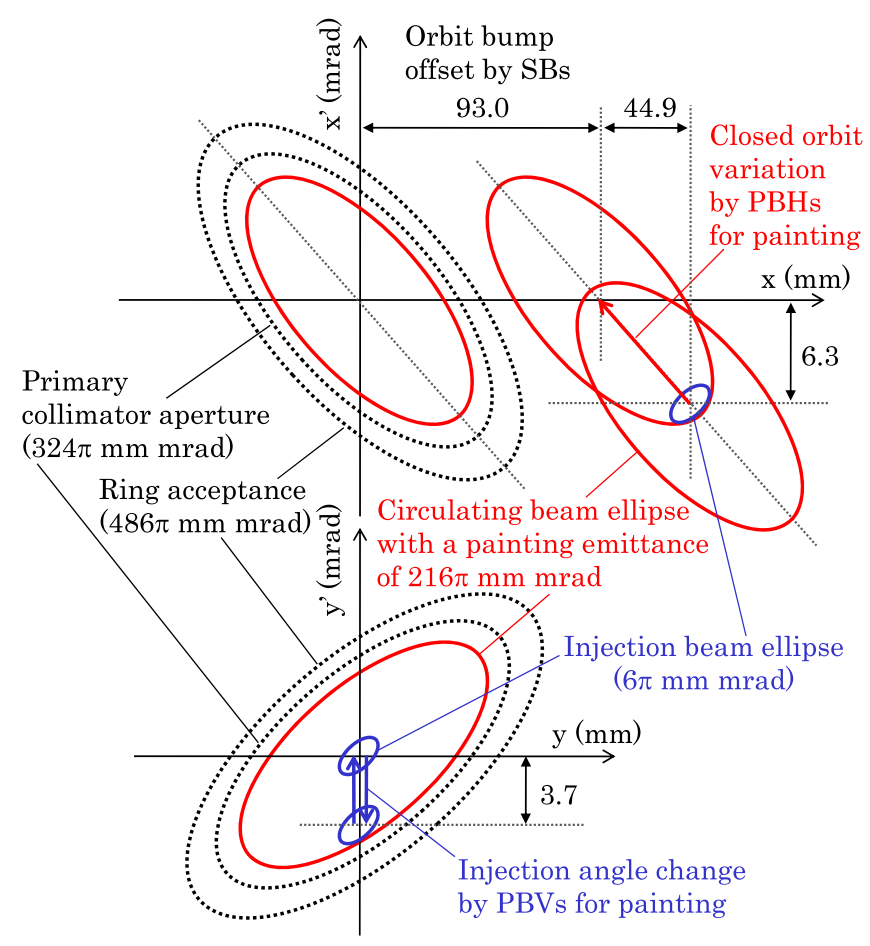

FIG. 4. Schematic view of the injection painting in the transverse plane, where the upper one is for the horizontal phase space, while the lower one is for the vertical one. 
single-particle behavior. On the other hand, another combination of (1) and (3) is known as anticorrelated painting. In this case, the transverse beam emittances are distributed along $\epsilon_{x}+\epsilon_{y} \approx$ const, producing an elliptical cross section of the circulating beam after painting, that is, forming a Kapchinskij-Vladimirskij-like distribution in theory. Analytical expressions of the transverse phase-space painting are described in Refs. [10,11].

While the maximum design painting emittance is $216 \pi \mathrm{mm}$ mrad, corresponding to $\left(x_{\max }, x_{\max }^{\prime}\right)=(44.9 \mathrm{~mm}$, $6.3 \mathrm{mrad})$ and $\left(y_{\max }, y_{\max }^{\prime}\right)=(0.0 \mathrm{~mm}, 3.7 \mathrm{mrad})$, it can be adjusted by altering the top of the excitation waveforms of the PBHs and PBVs. In the present beam tuning experiment, the correlated painting with a painting emittance of $100 \pi \mathrm{mm}$ mrad, corresponding to $\left(x_{\max }, x_{\max }^{\prime}\right)=(27.1 \mathrm{~mm}$, $3.8 \mathrm{mrad})$ and $\left(y_{\max }, y_{\max }^{\prime}\right)=(0.0 \mathrm{~mm}, 2.3 \mathrm{mrad})$, was mainly applied.

In this paper, the effectiveness of this correlated painting for beam loss reduction in combination with the longitudinal painting are discussed.

Details of the beam-based adjustment of the excitation waveforms of the PBHs and PBVs are described in Refs. [12,13].

\section{B. Longitudinal phase-space painting}

The longitudinal painting makes use of a controlled momentum offset to the rf bucket in combination with superposing a second harmonic rf voltage to obtain a uniform particle distribution in the longitudinal direction after the multiturn injection.

Figure 5 shows a schematic view of the momentum offset injection scheme. In the RCS, the momentum offset is realized by adding a frequency offset to the original rf frequency pattern. As shown in the right side of Fig. 5,
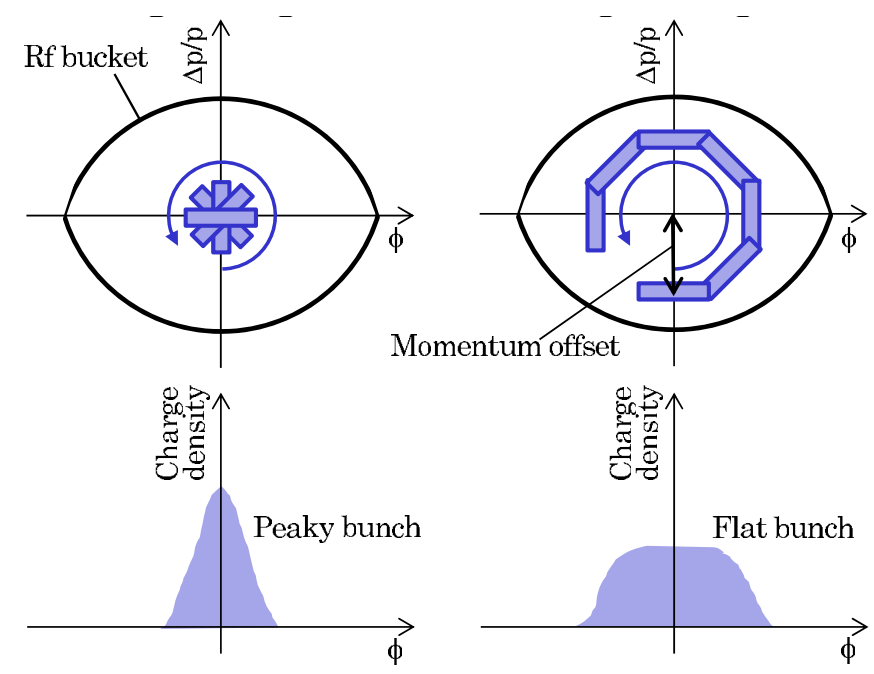

FIG. 5. Schematic view of the longitudinal motion for the multiturn injection process without (left) and with (right) a momentum offset, where the boxes represent the injection bunch train from the linac. a flat beam bunch is formed through emittance dilution by large synchrotron motion excited by the momentum offset. In this method, the superposition of the second harmonic rf voltage fills the role of shaping a flatter and wider rf bucket potential (compare the blue solid and red solid curves in Fig. 7 given later), which leads to better longitudinal motion to make a flatter bunch distribution. As an additional knob in the longitudinal painting, a phase sweep of the second harmonic rf voltage relative to the fundamental one was applied. This second harmonic phase sweep method enables further bunch distribution control through a dynamical change of the rf bucket potential shape including a position change of the stable fixed points during injection (compare the red curves in Fig. 7 given later).

The rf voltage $V_{\text {rf }}$ applied in the present experiment is expressed as

$$
V_{\mathrm{rf}}=V_{1} \sin \phi-V_{2} \sin \left\{2\left(\phi-\phi_{s}\right)+\phi_{2}\right\},
$$

where $V_{1}$ is the amplitude of the fundamental rf voltage, $V_{2}$ the amplitude of the second harmonic rf component, $\phi_{s}$ the synchronous phase, $\phi$ the phase of the rf voltage, and $\phi_{2}$ the phase offset of the second harmonic rf voltage. For improving the longitudinal beam distribution, the longitudinal motion was thoroughly surveyed for various combinations of rf parameters, especially $V_{2} / V_{1}, \phi_{2}$, and the momentum offset $\Delta p / p$, in both numerical simulations [14] and experiments [15]. In the present beam tuning experiment, the second harmonic rf voltage was employed typically with an amplitude of $80 \%$ of the fundamental one during the first $1 \mathrm{~ms}$, decreasing to $0 \mathrm{kV}$ in the next $2 \mathrm{~ms}$, as shown in Fig. 6. Also its phase was linearly swept from -100 to 0 degrees over an injection duration of $0.5 \mathrm{~ms}$. In this case, the shape of the rf bucket potential gradually changes during injection, as shown by the red curves in

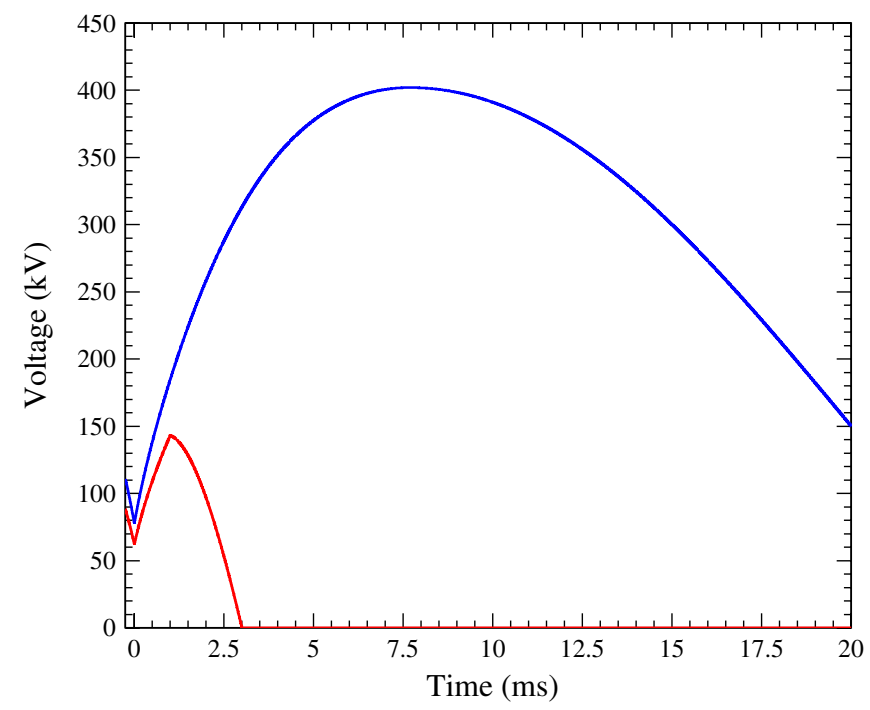

FIG. 6. Fundamental (blue) and second harmonic (red) rf voltage patterns over the acceleration period typically programmed in the present beam tuning experiment. 


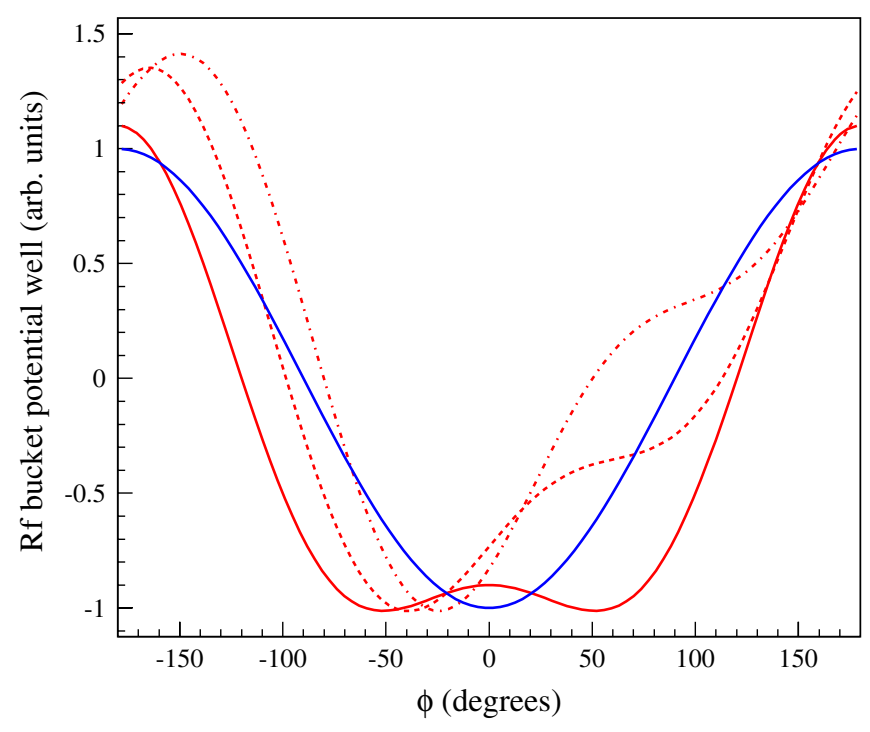

FIG. 7. The rf bucket potential well calculated with an amplitude ratio $V_{2} / V_{1}$ of $80 \%$ and a phase offset $\phi_{2}$ of 0 (red solid), -50 (red dashed), and -100 (red dash-dotted) degrees. In this figure, the potential well in the case of the only fundamental rf voltage is plotted as a blue solid curve for reference.

Fig. 7. By combining such a dynamically controlled $\mathrm{rf}$ bucket potential and the momentum offset injection of $-0.2 \%$ with the corresponding rf frequency offset, we obtained a well uniformly shaped longitudinal beam distribution, as is described in the following section (further details of the rf parameter dependence on the longitudinal motion are described in Refs. $[14,15])$.

In this paper, we investigate the influence of the longitudinal painting to the transverse motion, and the combined effect of the transverse and longitudinal painting in terms of beam loss reduction through space-charge mitigation.

\section{EXPERIMENTAL RESULTS AND DISCUSSIONS WITH NUMERICAL SIMULATIONS}

After performing the initial beam tuning and a series of underlying beam studies with low-intensity beams to examine the basic lattice properties [2], we have been conducting beam tuning experiments with high-intensity beams including the injection painting scheme since December 2009. In this section, the results of a highintensity trial experiment performed in January 2011 are mainly described together with the corresponding numerical simulation results.

\section{A. Experimental conditions}

This experiment was performed in a single-shot beamon-demand operation using a $0.5 \mathrm{~ms}$-long linac pulse with a peak current of $20 \mathrm{~mA}$ and a chopper beam-on duty factor of $56 \%$. In this case, the number of particles per pulse totals $3.5 \times 10^{13}$, corresponding to $420 \mathrm{~kW}$ output beam power if it is running at the design repetition rate of $25 \mathrm{~Hz}$. For this injection beam, we attempted to reduce beam loss arising from the space-charge effect in the low-energy region by employing the injection painting technique, where the horizontal and vertical tunes were chosen at $(6.45,6.42)$ to avoid the possible effects of strong betatron resonances.

The injection painting parameters tested in this beam tuning experiment are listed in Table II. The correlated painting with a painting emittance $\left(\epsilon_{t p}\right)$ of $100 \pi \mathrm{mm} \mathrm{mrad}$ was mainly applied for the transverse painting. On the other hand, the rf parameters mentioned in the previous section were used for the longitudinal painting. That is, a second harmonic rf voltage with an amplitude of $80 \%$ of the fundamental one $\left(V_{1} / V_{2}\right)$ and its phase sweep $\left(\phi_{2}\right)$ from -100 to 0 degrees were employed, for which a momentum offset injection $(\Delta p / p)$ of 0 to $-0.2 \%$ was tested.

With systematic combinations of the transverse and longitudinal painting parameters in Table II, we surveyed their effectiveness for beam loss reduction.

\section{B. Numerical simulation setup}

The corresponding numerical simulation was performed with a particle-tracking code called SIMPSONS [16] developed by Shinji Machida. This code enables us to simulate 3-dimensional motion of beam particles including the injection painting process.

TABLE II. Injection painting parameters applied in the present beam tuning experiment: ID 1, no painting (center injection); ID 2, transverse painting only; IDs 3-5, longitudinal painting only; and IDs $6-8$, combinations of transverse and longitudinal painting.

\begin{tabular}{lcccc}
\hline \hline Parameter ID & $\epsilon_{t p}(\pi \mathrm{mm}$ mrad $)$ & $V_{2} / V_{1}(\%)$ & $\phi_{2}($ degrees $)$ & $\Delta p / p(\%)$ \\
\hline 1 & $\ldots$ & $\ldots$ & $\ldots$ & $\ldots$ \\
2 & 100 [correlated painting] & $\ldots$ & $\ldots$ & $\ldots$ \\
3 & $\ldots$ & 80 & -100 & $\ldots$ \\
4 & $\ldots$ & 80 & -100 & -0.1 \\
5 & $\ldots$ & 80 & -100 & -0.2 \\
6 & 100 [correlated painting] & 80 & -100 & $\ldots$ \\
7 & 100 [correlated painting] & 80 & -100 & -0.1 \\
8 & 100 [correlated painting] & 80 & -100 & -0.2 \\
\hline \hline
\end{tabular}


In the SIMPSONS, all the lattice elements are represented as thin lens elements. The conversion from the thick lens to the thin lens description is performed with another code TEAPOT [17]. In this stage, tune fitting and lattice imperfections such as field and alignment errors are reflected in the thin lens representation. In the present simulation, all the lattice imperfections, which have been measured so far, were included; multipole field components of all the ring magnets [18,19], magnetic field errors [20], misalignment, static leakage fields from the extraction beam-line magnets, and edge focus of the injection-orbit-bump magnets [21].

The independent variable of the SIMPSONS is time, not the longitudinal position. That is very useful in calculating the space-charge potential, since a snapshot of the beam in the configuration space is directly obtained. In the SIMPSONS, the particle-in-cell algorithm with cylindrical meshes in the $(r, \theta, z)$ coordinates is used to compute the space-charge potential. The fractional charge of each macroparticle is assigned to the nearby grid points following the area-weighting method. The charge distribution at the grid points is Fourier transformed in the azimuthal direction, and then the Poisson equation is solved in the $(r, z)$ space for each azimuthal mode, assuming a boundary condition of the circular cross sectional beam pipe made of perfectly conducting material. Finally, the space-charge force is applied to each macroparticle as a 3-dimensional impulse kick. The space-charge potential is recalculated at a certain time step out of the macroparticles whose distribution evolves in a self-consistent manner as per the progression of time. The time step applied in the present simulation was 2 ns during injection and $5 \mathrm{~ns}$ for the others, corresponding to 1000 and 400 kicks per turn.

In the present numerical simulation, the number of macroparticles was chosen to be $2 \times 10^{5}$ using a transverse grid of $64(r) \times 64(\theta)$ for the conducting boundary of $r=$ $0.17 \mathrm{~m}$, and a longitudinal grid of $100(z)$. This number of macroparticles was kept constant during multiturn injection by adjusting charge per macroparticle, to obtain more reliable simulation results in the injection painting process. Figures 8 and 9 show the macroparticle number dependences of the numerical simulation results obtained for the painting parameter ID 8 in Table II. As is evident in the figures, the behavior of the transverse emittance growth for the edge part of the beam as well as that of the beam loss show a trend to converge for $2 \times 10^{5}$ macroparticles or more. Those signify the use of $2 \times 10^{5}$ macroparticles is tolerable to obtain meaningful simulation results in the present work. As is mentioned in the following subsection, in fact, the present numerical simulation well reproduced the painting parameter dependence of the beam loss rate measured in the range of $1 \times 10^{-2}-2 \times 10^{-1}$. These numerical parameters are tolerable also in terms of getting acceptable CPU time.

The RCS involves the beam loss arising from large angle events scattered on the stripping foil during injection. Such an effect was included in the SIMPSONS by using the scattering angle distribution calculated with GEANT [22] assuming both Coulomb and hadronic interactions beforehand.

High-intensity beam accelerations generally involve an rf bucket distortion arising from the beam loading, while, in the present numerical simulation, such an effect is not included. In this experiment, however, the beam loading effect was well compensated by the multiharmonic if feedforward method [23]. Therefore, the beam then felt the accelerating $\mathrm{rf}$ voltage as programmed. This beam loading compensation not only gives better longitudinal motions for high-intensity beams, but provides a more reliable comparison of the present numerical simulation and the measurement.

\section{Beam loss}

The left plot in Fig. 10 shows the beam survival rate (defined as the ratio of the output and input intensities) evaluated from a signal ratio of a dc current transformer (DCCT) installed in the ring and a slow current transformer (SCT) installed in the linac section, where the DCCT signal at the extraction time is used as the output intensity. On the other hand, the right plot in Fig. 10 shows the output beam intensity measured with an SCT installed in the 3-NBT. The horizontal axes here correspond to the injection painting parameter ID numbers in Table II. As shown by the data ID 1 in the figure, the beam survival rate in the case of no painting was measured to be $83 \%$, where the injected beam was accumulated at the center region in
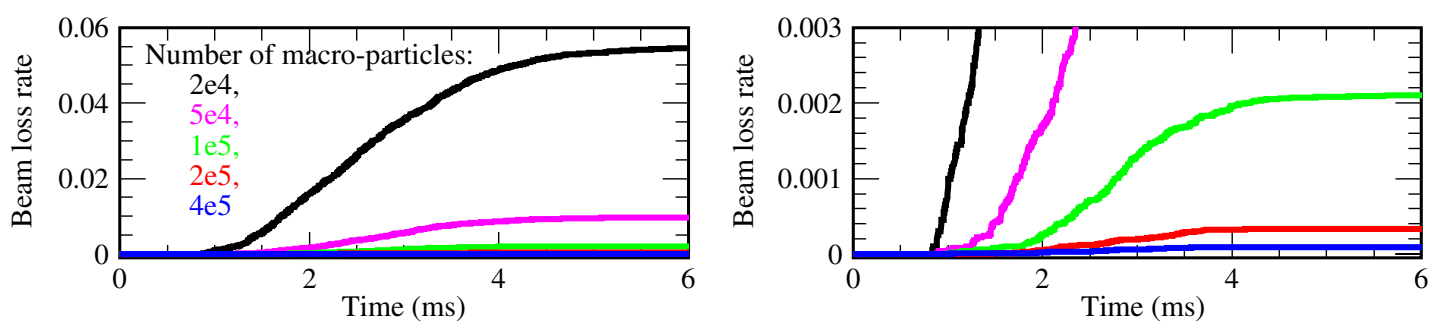

FIG. 8. Macroparticle number dependence (black, $2 \times 10^{4}$; pink, $5 \times 10^{4}$; green, $1 \times 10^{5}$; red, $2 \times 10^{5}$; and blue, $4 \times 10^{5}$ ) of the beam loss calculated for the first $6 \mathrm{~ms}$ with the painting parameter ID 8 in Table II, where the left and right plots have different ranges in the vertical axis. In this calculation, no foil scattering was included. 

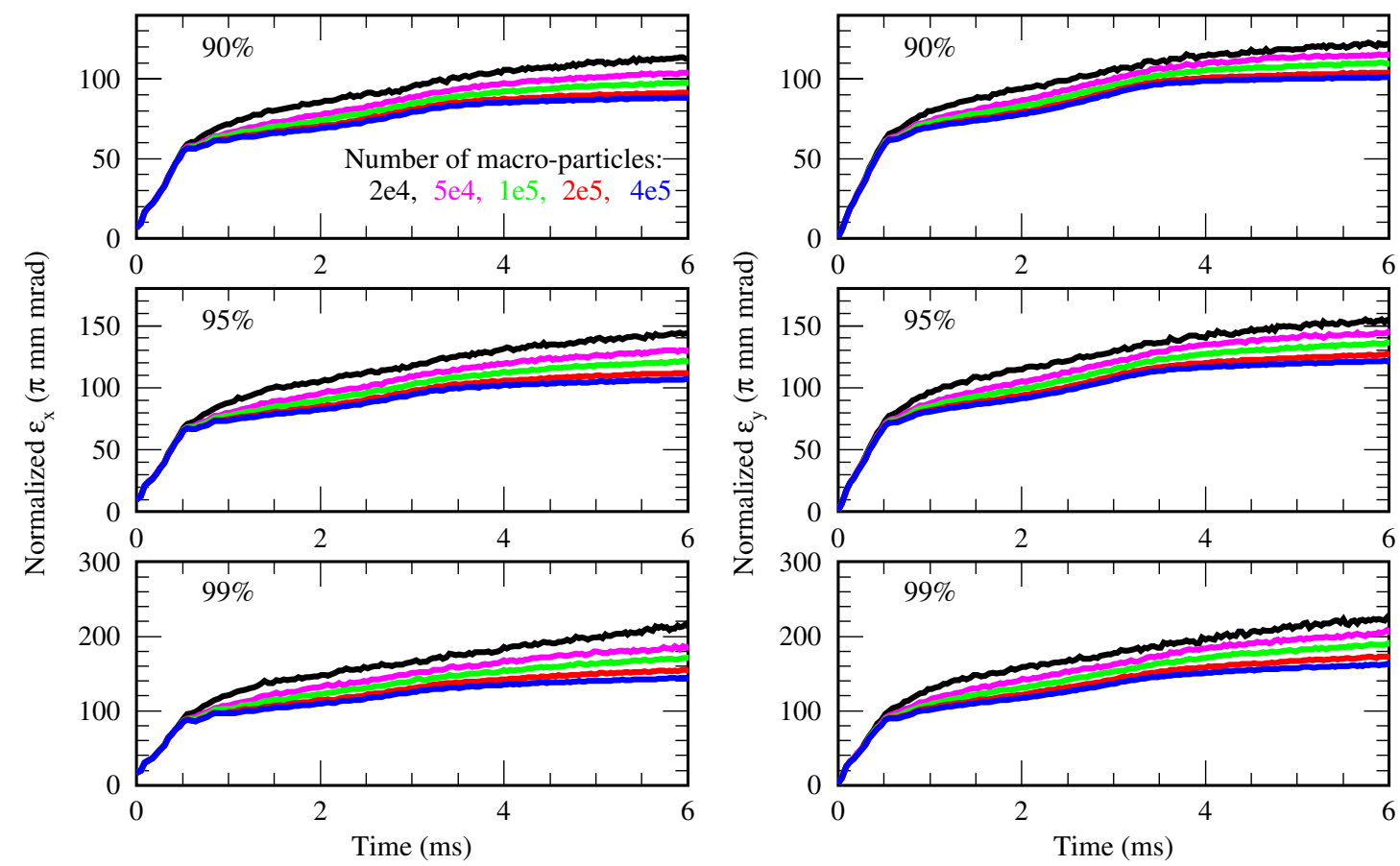

FIG. 9. Macroparticle number dependence (black, $2 \times 10^{4}$; pink, $5 \times 10^{4}$; green, $1 \times 10^{5}$; red, $2 \times 10^{5}$; and blue, $4 \times 10^{5}$ ) of the horizontal (left) and vertical (right) normalized emittances calculated for the first $6 \mathrm{~ms}$ with the painting parameter ID 8 in Table II, where different emittances encircling $90 \%, 95 \%$, and $99 \%$ of the macroparticles are plotted. In this calculation, no foil scattering was included.
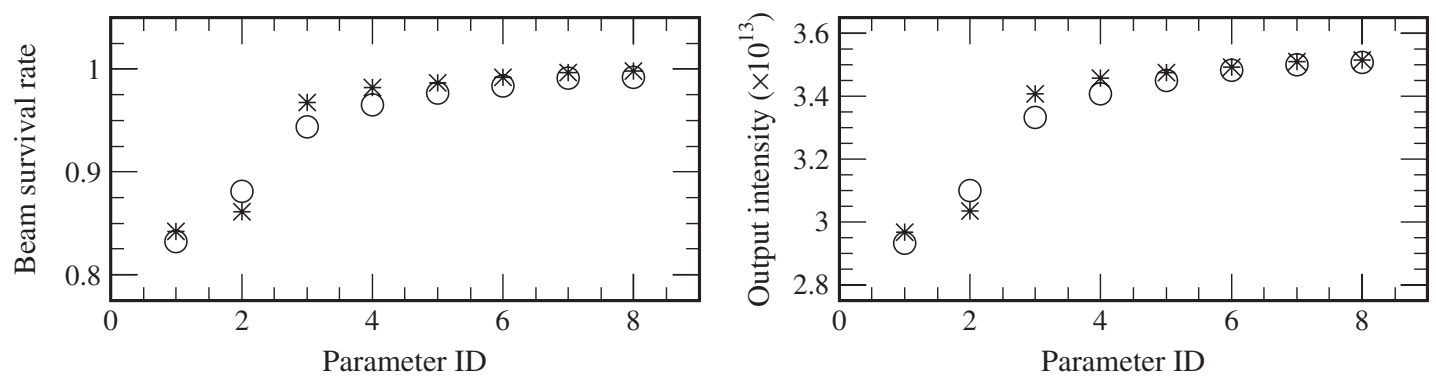

FIG. 10. Beam survival rate (defined as the ratio of the output and input intensities) evaluated from a signal ratio of the DCCT installed in the ring and the SCT installed in the linac section (left), and the corresponding output intensity (the number of particles per pulse) measured with the SCT installed in the 3-NBT (right), where the horizontal axes correspond to the painting parameter ID numbers in Table II. In the figure, the corresponding simulated result is plotted as a star.

the transverse and longitudinal phase spaces with no charge density manipulation (called center injection). In order to reduce the observed $17 \%$ intensity loss, we introduced injection painting. As shown in the figure, the beam survival rate gradually improved from the data IDs 1 to 8 by introducing the transverse painting, the longitudinal painting and their combinations. The best beam survival rate obtained for the parameter ID 8 was better than $99 \%$, where the highest output intensity, corresponding to $420 \mathrm{~kW}$ output beam power, was obtained. In this figure, the stars represent the corresponding simulated results. The painting parameter dependence of the simulated beam survivals is in good agreement with that of the measured ones.
Figure 11 shows the time dependence of the circulating beam intensity from injection through extraction, measured with the DCCT installed in the ring, where the ramp-up slope of the beam intensity from 0 to $0.5 \mathrm{~ms}$ corresponds to the beam accumulation process. In this figure, we can see that the beam loss appears only for the first several $\mathrm{ms}$ in the low-energy region, where the spacecharge effect is the most serious. As the injection painting progresses, the observed intensity loss is gradually reduced, and mostly recovered for the parameter ID 8 . The measured intensity curve for the parameter ID 8 is nearly flat after the injection process. Particularly in this measurement, we confirmed a powerful ability of the longitudinal painting for beam loss reduction. In addition, Fig. 11 shows 

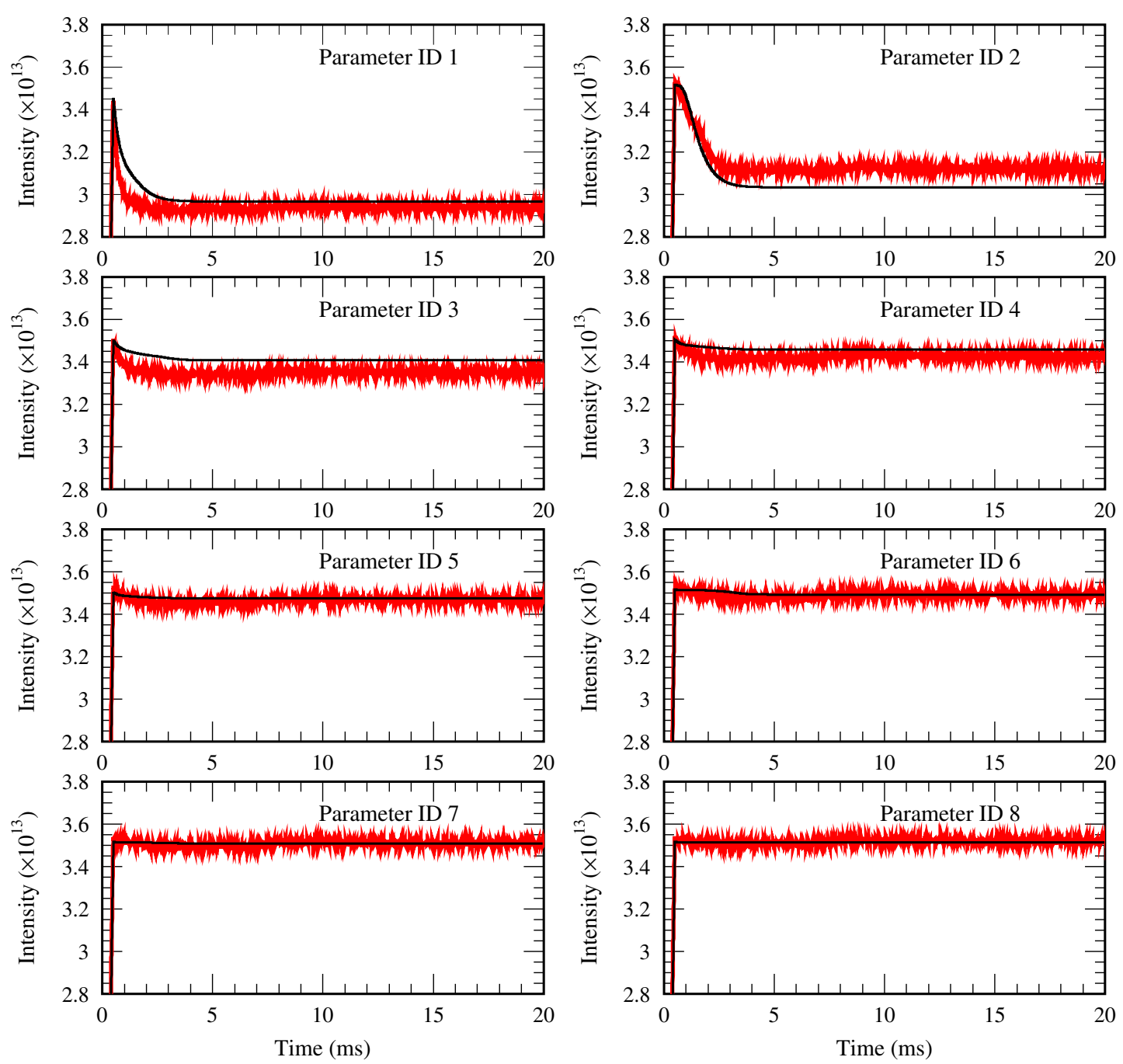

FIG. 11. Time dependence of the circulating beam intensity from injection through extraction, measured with the DCCT installed in the ring, where the ramp-up slope of the beam intensity from 0 to $0.5 \mathrm{~ms}$ corresponds to the beam accumulation process. In the figure, the corresponding simulated result is plotted as a black solid curve.

that the intensity peaks at the end of injection $(0.5 \mathrm{~ms})$ vary slightly with the injection painting parameters. As is subsequently discussed, this result reflects the beam loss mainly caused by the foil scattering during beam accumulation. The corresponding simulated results, plotted as black solid curves in the figure, well reproduce the measured intensity loss patterns.

In order to investigate the time dependence of the beam loss in more detail, we employed a scintillation-type beam loss monitor (BLM) with a photomultiplier, because the use of this BLM helps in obtaining excellent time response and resolution. The top plot in Fig. 12 shows the time structure of the scintillation-type BLM signal for the first $6 \mathrm{~ms}$ measured in the collimator section for the data IDs 3 to 8, while the middle plot in Fig. 12 shows the corresponding simulated result plotted as the number of lost macroparticles per turn. Integrated curves of the beam loss distributions in the top and middle plots are compared in the bottom plot in Fig. 12, where the simulated curves are normalized as the beam loss rate, and the measured curves are scaled to fit the simulated patterns. The measured and simulated beam loss patterns are in good agreement also in this case. In these figures, the contribution of the transverse painting to beam loss reduction, in addition to that of the longitudinal painting, can be clearly observed; by comparing the left (parameter IDs 3-5) and right (parameter IDs 6-8) plots in Fig. 12, it is confirmed that the transverse painting effectively decreases the beam loss mainly for the first $1 \mathrm{~ms}$ region. The beam loss in this period includes a component arising from the foil scattering during the charge-exchange injection process, or more precisely, while the injection-orbit bump is active. The foil scattering beam loss is essentially proportional to the foil-hitting probability during injection. While the longitudinal painting hardly changes the number of foil hits during injection, the transverse painting reduces this number, because of the 

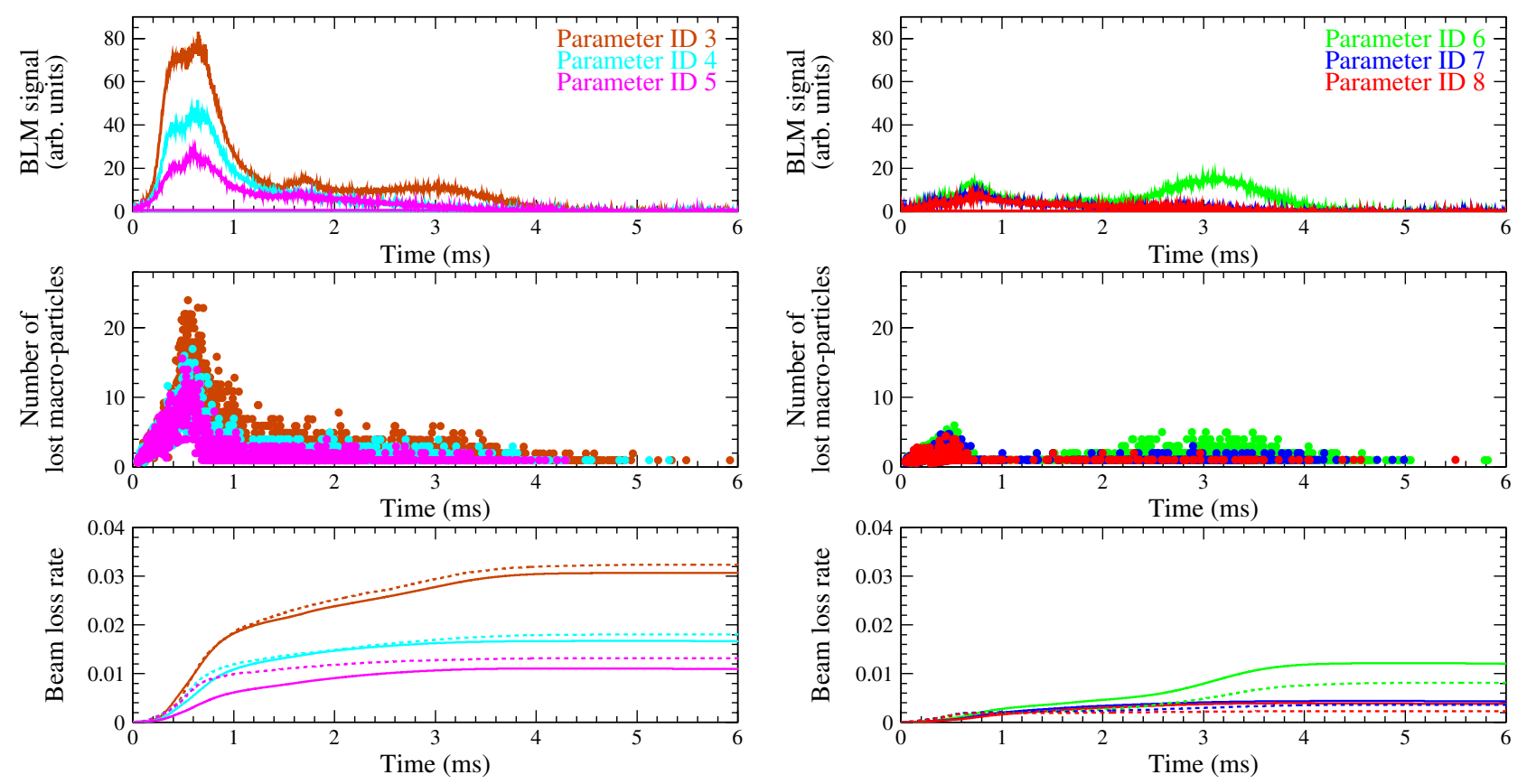

FIG. 12. Time structure of the scintillation-type BLM signal for the first $6 \mathrm{~ms}$ measured in the collimator section for the parameter IDs 3 (brown), 4 (light blue), 5 (pink), 6 (green), 7 (blue), and 8 (red) in Table II (top), and the corresponding simulated result plotted as the number of lost macroparticles per turn (middle). Integrated curves of the measured and simulated beam loss distributions in the top and middle plots are compared in the bottom plot, where the simulated curves (dashed) are normalized as the beam loss rate, and the measured curves (solid) are scaled to fit the simulated patterns.

horizontal closed orbit moving away from the foil and the excited betatron amplitudes larger than the foil size. By this case of transverse painting, the number of foil hits per particle during injection is reduced from 90 to 24. Therefore, the beam loss reduction by the transverse painting observed in Fig. 12 includes the effect of the foilhitting rate reduction as well as that of the charge density control. In order to distinguish between the two contribu- tions, we performed additional numerical simulations with no foil scattering. The upper plot in Fig. 13 shows the beam loss distribution simulated with no foil scattering for the data IDs 3 to 8 , and the lower plot shows the comparison of their integrated curves with the simulated curves including the foil scattering given in Fig. 12. By comparing the left and right sides of Fig. 13, we can discretely confirm the significant beam loss reduction by the charge density
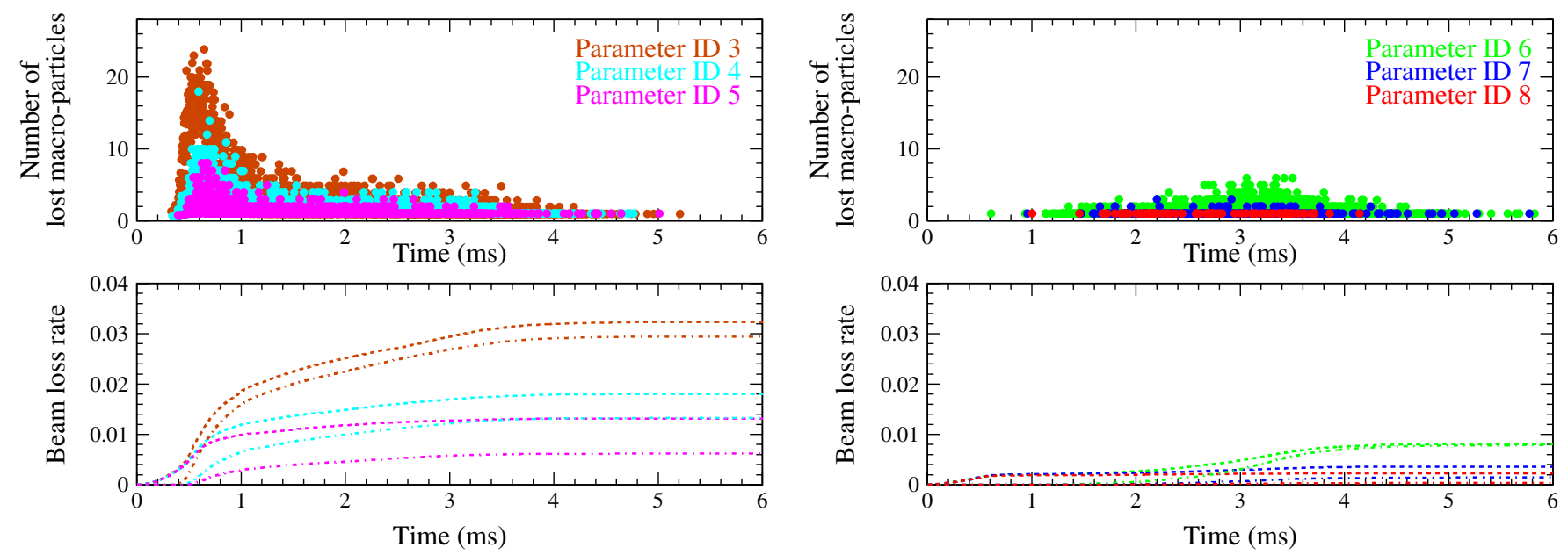

FIG. 13. Beam loss distribution simulated with no foil scattering plotted as the number of lost macroparticles per turn for the parameter IDs 3 (brown), 4 (light blue), 5 (pink), 6 (green), 7 (blue), and 8 (red) in Table II (top). Their integrated curves (dash dotted) are compared in the bottom plot with the simulated results including the foil scattering (dashed) given in Fig. 12. In the bottom plot, the difference of the dashed and dash-dotted curves corresponds to the beam loss component arising from the foil scattering. 

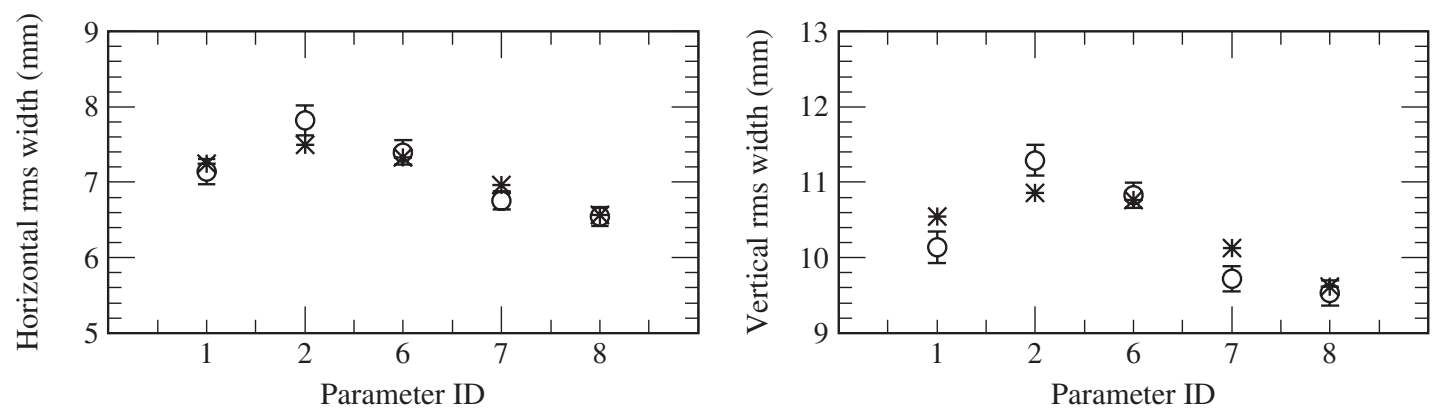

FIG. 14. Painting parameter dependence of the horizontal (left) and vertical (right) rms widths of the extracted beam, measured with the MWPM installed in the 3-NBT, where the horizontal axis shows the painting parameter ID numbers in Table II. In the figure, the corresponding simulated results are plotted as stars.
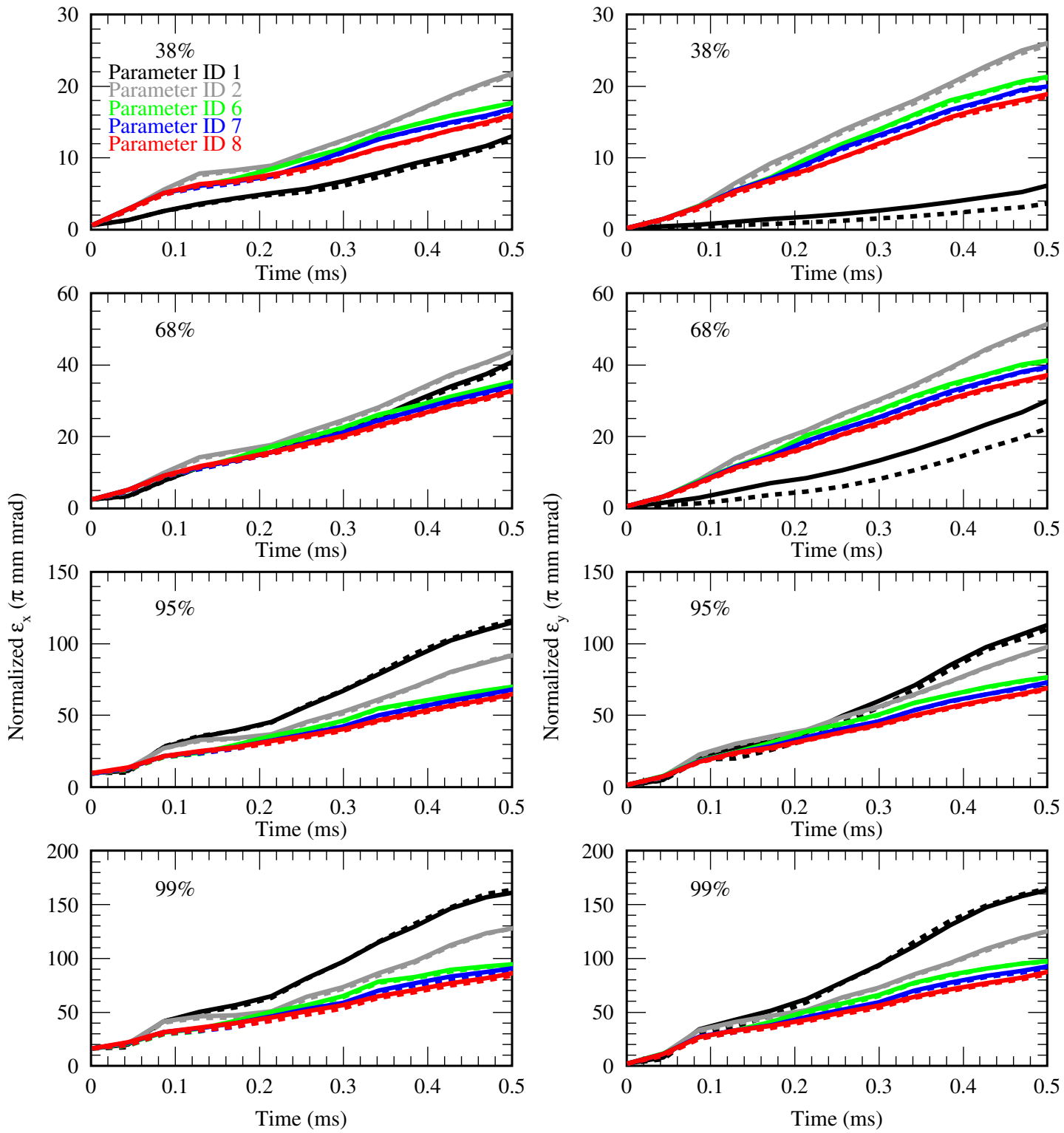

FIG. 15. Time evolution of the horizontal (left) and vertical (right) normalized emittances over an injection duration of $0.5 \mathrm{~ms}$ simulated for the parameter IDs 1 (black), 2 (gray), 6 (green), 7 (blue), and 8 (red), where different emittances encircling 38\%, 68\%, $95 \%$, and $99 \%$ of the macroparticles are plotted for each parameter ID. In the figure, similar results simulated with no foil scattering are also plotted as dashed curves for reference. 

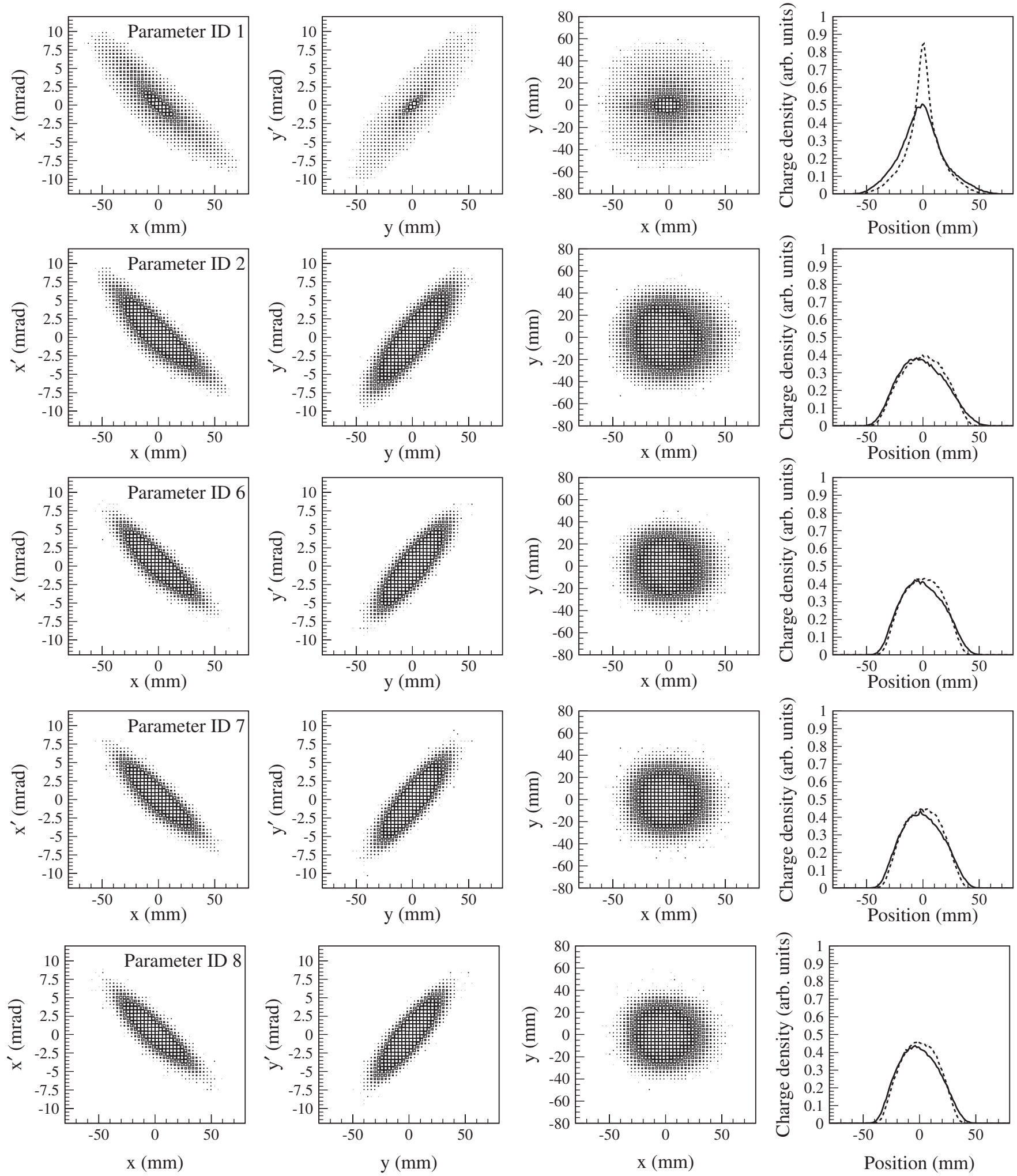

FIG. 16. Simulated 2-dimensional plots of $\left(x, x^{\prime}\right),\left(y, y^{\prime}\right)$, and $(x, y)$ spaces at the end of injection on the foil and their projections to the $x$ and $y$ axes for the parameter IDs $1,2,6,7$, and 8, where in the far right plots the solid and dashed curves correspond to the horizontal and vertical profiles. 
control in the transverse painting, in addition to that by its resultant decrease in the foil-hitting rate.

As shown in Figs. 10-12, the series of numerical simulations as a whole well reproduce the beam loss patterns observed for several independent measurements. The remaining small beam loss of less than $1 \%$ obtained in the simulation with the painting parameter ID 8 is mainly caused by the foil scattering during the charge-exchange process (see the red dashed and red dash-dotted curves in the lower right plot in Fig. 13). That is, the simulation implies that the space-charge induced beam loss for the $420 \mathrm{~kW}$-equivalent intensity beam is mostly minimized through the charge density control in the transverse and longitudinal painting with the parameter ID 8 .

\section{Beam distribution}

Figure 14 shows the painting parameter dependence of the horizontal and vertical rms widths of the extracted beam, measured with a multiwire profile monitor (MWPM) installed in the 3-NBT for the parameter IDs 1, $2,6,7$, and 8. In this figure the stars represent the corresponding simulated results, which are in good agreement with the measured ones. As shown in the figure, the rms width of the extraction beam increases slightly by the transverse painting (parameter IDs 1 to 2), but gradually decreases with the progression of longitudinal painting (parameter IDs 2 to 8). Such a state is led through the basic functions of the transverse and longitudinal painting, as is mentioned below.

Figure 15 shows the time evolution of the horizontal and vertical normalized emittances over an injection duration of 0.5 ms simulated for the parameter IDs $1,2,6,7$, and 8 , where different emittances encircling 38\%, 68\%, 95\%, and $99 \%$ of the macroparticles are plotted for each parameter ID. In the figure, similar results simulated for the case with no foil scattering are also plotted as dashed curves for reference. In this figure, we can see some contribution of the foil scattering to emittance growth for the data ID 1; this is illustrated as the difference of the black solid and dashed curves. On the other hand, in the data IDs 2 to 8 , this contribution is negligible, because the transverse painting decreases the foil-hitting probability during injection. In addition, this figure shows the characteristic features of the transverse and longitudinal painting. As shown by the emittance change from the data IDs 1 to 2 , the transverse painting increases the beam emittances for $38 \%$ and $68 \%$, while it decreases those for $95 \%$ and $99 \%$. On the other hand, the emittance variations from the data IDs 2 toward 8 show that the longitudinal painting acts to decrease all the 38\%-99\% emittances.

Such a characteristic aspect is more directly observed in Fig. 16, which shows the simulated 2-dimensional plots of $\left(x, x^{\prime}\right),\left(y, y^{\prime}\right)$, and $(x, y)$ spaces at the end of injection on the foil and their projections to the $x$ and $y$ axes for the parameter IDs $1,2,6,7$, and 8 . In order to clarify the transverse charge distribution change by the transverse and longitudinal painting, the differences in the simulated transverse distributions for the parameter IDs 1 and 2, and for the parameter IDs 2 and 8 are plotted in Fig. 17. In the transverse painting, the beam is widely distributed directly in the transverse phase space following the painting functional form. Therefore, the basic function of the
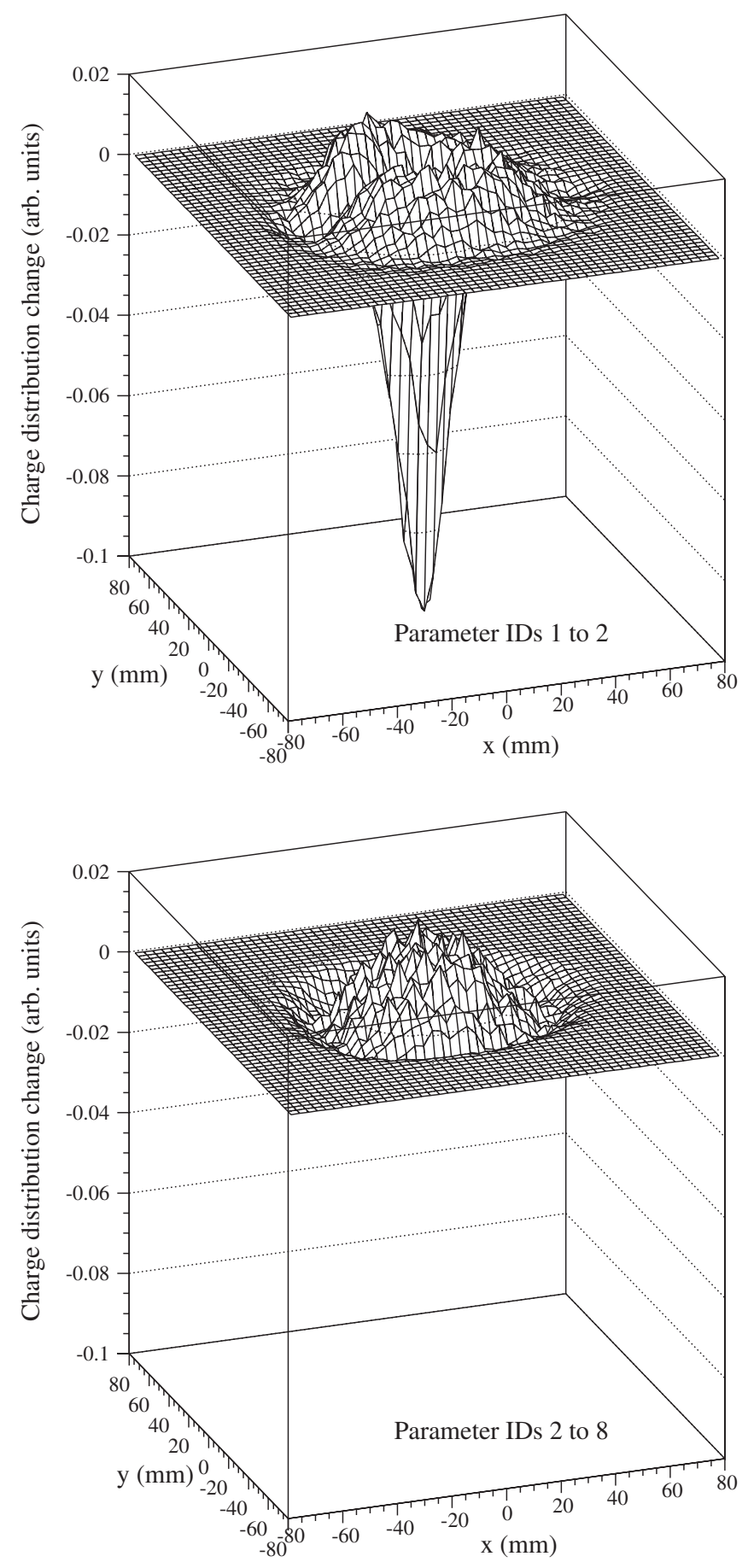

FIG. 17. Differences in the simulated 2-dimensional $(x, y)$ plots for the parameter IDs 1 and 2 (top), and for the parameter IDs 2 and 8 (bottom) given in Fig. 16. 

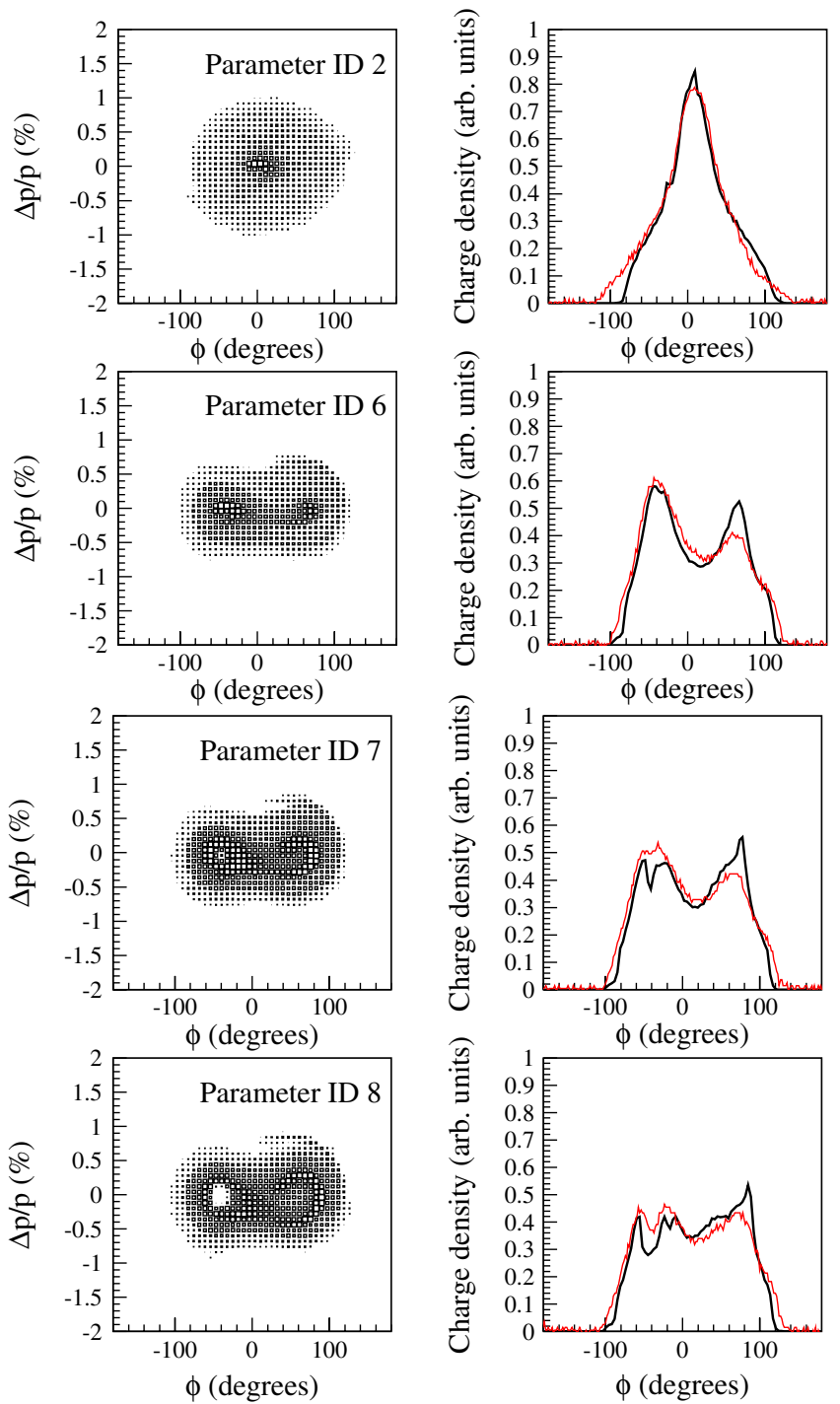

FIG. 18. Simulated 2-dimensional plot of the longitudinal phase space $(\phi, \Delta p / p)$ at the end of injection and its projection to the $\phi$ axis for the parameter IDs 2, 6, 7, and 8, where the red solid curve in the projection plot shows the corresponding distribution measured with the WCM.

transverse painting is to widen the core part of the beam and to decrease the tail. For the transverse distribution change from the parameter IDs 1 to 2 in Fig. 17, such a feature in the transverse painting is evident; that is, beam halo or beam tail reduction by a decrease in the peak density of the transverse distribution is observed. On the other hand, the longitudinal painting acts to shrink the entire transverse beam distribution, as shown for the transverse distribution change from the parameter IDs 2 to 8 in Fig. 17. Figure 18 shows the simulated 2-dimensional plot of the longitudinal phase space $(\phi, \Delta p / p)$ at the end of injection and its projection to the $\phi$ axis for the parameter IDs $2,6,7$, and 8 . In this figure, the corresponding longitudinal beam profile measured with a wall current monitor (WCM) is also plotted as a red solid curve, which the simulated profile well reproduces. As is evident in the figure, the flatness level of the beam bunch significantly improves with the progression of the longitudinal painting. The bunching factor (defined as the ratio of the average current to the peak current of the circulating beam) improves from 0.25 (parameter ID 2) to 0.45 (parameter ID 8) at the end of injection. The transverse beam size reduction including both the core and tail parts observed in the lower plot in Fig. 17 is led through such a charge density mitigation in the longitudinal direction.

The above analyses for simulated particle distributions indicate that the significant beam loss reduction observed in Figs. 10-12 is led through the beam halo/tail reduction by the charge density reduction in the transverse and longitudinal directions and its resultant space-charge mitigation.

\section{SUMMARY}

In the J-PARC 3-GeV RCS, we performed a highintensity beam trial with the injection painting technique in January 2011 using a $0.5 \mathrm{~ms}$-long linac pulse with a peak current of $20 \mathrm{~mA}$ and a chopper beam-on duty factor of $56 \%$. The number of particles per pulse totaled $3.5 \times 10^{13}$, corresponding to $420 \mathrm{~kW}$ output beam power if running at $25 \mathrm{~Hz}$. In this experiment, we confirmed a powerful ability of the injection painting for beam loss reduction, and successfully accomplished the high-intensity demonstration at a low-level intensity loss of less than $1 \%$ by the combination of the transverse and longitudinal painting.

The corresponding numerical simulation shows that the present transverse and longitudinal painting effectively suppresses the space-charge induced beam halo/tail formation through charge density reduction in the transverse and longitudinal directions and it leads to the observed significant beam loss reduction. In addition, the simulation shows that the remaining small beam loss of less than $1 \%$ mostly arises from the foil scattering during the chargeexchange injection process. In other words, it signifies that the space-charge induced beam loss for the $420 \mathrm{~kW}$ equivalent intensity beam is mostly minimized by the present injection painting. The present numerical simulation well reproduced the empirical painting parameter dependence on the beam loss and beam profile, and assisted our understanding of the characteristic behavior of the high-intensity beam in the transverse and longitudinal painting process.

The low-loss beam operation for the $420 \mathrm{~kW}$-equivalent intensity beam achieved in this experiment is considered to be a credible large step toward realizing our final goal of 1 MW output, because the space-charge effect at the current injection energy of $181 \mathrm{MeV}$ in this experiment is more severe than that at the higher injection energy of $400 \mathrm{MeV}$ in the $1 \mathrm{MW}$ beam operation.

Further efforts will be directed at understanding highintensity beam behavior, with focus on more detailed 
mechanisms underlying the observed beam loss and beam loss reduction.

\section{ACKNOWLEDGMENTS}

The authors would like to acknowledge all members of the J-PARC RCS, linac, MR, and MLF groups for their indispensable support during the present beam tuning experiment. We are also grateful to Professor E. Takasaki, Professor A. Ando, Professor Y. Yamazaki, and Dr. S. Machida for their valuable advice and fruitful discussions.

[1] High-intensity Proton Accelerator Project Team, JAERI Report No. JAERI-Tech 2003-044 and KEK Report No. 2002-13.

[2] H. Hotchi et al., Phys. Rev. ST Accel. Beams 12, 040402 (2009).

[3] J. Beebe-Wang, Y. Y. Lee, D. Raparia, and J. Wei, in Proceedings of the 1999 Particle Accelerator Conference, New York (IEEE, New York, 1999), pp. 1743-1745.

[4] J. Beebe-Wang, in Proceedings of the 1999 Particle Accelerator Conference, New York (Ref. [3]), pp. 2843-2845.

[5] B. Jones, D. Adams, and C. Warsop, in Proceedings of the 2007 Particle Accelerator Conference, Albuquerque, New Mexico (IEEE, New York, 2007), pp. 1640-1642.

[6] A. Seville, I. Gardner, J. Thomason, D. Adams, D. Bayley, and C. Worsop, in Proceedings of the 11th European Particle Accelerator Conference, Genoa, 2008 (EPS-AG, Genoa, Italy, 2008), pp. 349-351.

[7] I. Sugai, Y. Takeda, M. Oyaizu, H. Kawakami, Y. Irie, A. Takagi, H. Hattori, and K. Kawasaki, Nucl. Instrum. Methods Phys. Res., Sect. A 613, 457 (2010).

[8] H. Ao and Y. Yamazaki, Phys. Rev. ST Accel. Beams 15, 011001 (2012).

[9] Y. Yamazaki, in Proceedings of the 2009 Particle Accelerator Conference, Vancouver, BC, Canada (IEEE, Piscataway, NJ, 2009), pp. 18-22.
[10] Y. Kamiya, in Proceedings of the 1989 IEEE Particle Accelerator Conference, Chicago (IEEE, New York, 1989), pp. 660-662.

[11] J. Beebe-Wang, Y. Y. Lee, D. Raparia, J. Wei, C. R. Prior, and S. Machida, in Proceedings of the 2000 European Particle Accelerator Conference, Vienna, Austria (EPS, Geneva, 2000), pp. 1465-1467.

[12] P. K. Saha, Y. Shobuda, H. Hotchi, N. Hayashi, T. Takayanagi, H. Harada, and Y. Irie, Phys. Rev. ST Accel. Beams 12, 040403 (2009).

[13] H. Harada, Ph.D. thesis, Hiroshima University [KEK Report No. 2009-70, 2009].

[14] M. Yamamoto, M. Nomura, A. Schnase, T. Shimada, H. Suzuki, F. Tamura, E. Ezura, K. Hara, K. Hasegawa, C. Ohmori, K. Takata, A. Takagi, M. Toda, and M. Yoshii, Nucl. Instrum. Methods Phys. Res., Sect. A 621, 15 (2010).

[15] F. Tamura, M. Yamamoto, M. Yoshii, C. Ohmori, M. Nomura, A. Schnase, M. Toda, H. Suzuki, T. Shimada, K. Hara, and K. Hasegawa, Phys. Rev. ST Accel. Beams 12, 041001 (2009).

[16] S. Machida and M. Ikegami, AIP Conf. Proc. 448, 73 (1998).

[17] L. Schachinger and R. Talman, Part. Accel. 22, 35 (1987).

[18] H. Hotchi, F. Noda, N. Tani, J. Kishiro, S. Machida, and A. Yu. Molodojentsev, in Proceedings of the 2005 Particle Accelerator Conference, Knoxville, Tennessee (IEEE, Piscataway, NJ, 2005), pp. 916-918.

[19] H. Hotchi, F. Noda, Y. Irie, S. Machida, and A. Yu. Molodojentsev, in Proceedings of the 2006 European Particle Accelerator Conference, Edinburgh, UK (EPSAG, Edinburgh, Scotland, 2006), pp. 2104-2106.

[20] H. Hotchi and F. Noda, in Proceedings of the 3rd Annual Meeting of Particle Accelerator Society of Japan, Sendai, Japan, 2006, pp. 409-411.

[21] H. Hotchi, in Proceedings of the 2011 International Particle Accelerator Conference, San Sebastian, Spain (2011), pp. 6-10.

[22] See http://geant4.cern.ch.

[23] F. Tamura, M. Yamamoto, C. Ohmori, A. Schnase, M. Yoshii, M. Nomura, M. Toda, T. Shimada, K. Hara, and K. Hasegawa, Phys. Rev. ST Accel. Beams 14, 051004 (2011). 